\title{
PLANE SECTIONS THROUGH AN ASYMPTOTIC TANGENT OF A SURFACE
}

BY E. P. LANE

1. Introduction. It is well known that the plane curve in which an analytic surface $S$ in ordinary space is cut by a plane through an asymptotic tangent at an ordinary non-parabolic point $O$ of $S$ has an inflexion at $O$, provided that the plane is not the tangent plane of $S$ at $O$. Bompiani has enriched* the projective differential geometry of a plane curve in the neighborhood of an inflexion point on it by introducing various osculants covariantly associated with the inflexion. B. Su has applied $\dagger$ some of Bompiani's results in connection with the geometry of curves and surfaces in ordinary space. It is the purpose of this note to supplement the investigations of $\mathrm{Su}$, by studying further the loci of the osculants and various points and lines associated by Bompiani with the inflexion point of a plane curve of section of a surface made by a variable plane through a fixed asymptotic tangent of the surface at the point.

2. Power Series Expansions. G. M. Green calculated $\ddagger$ a canonical power series expansion for one non-homogeneous projective coordinate $z$ of a point on a surface in terms of the other two coordinates $x, y$. If the unit point for Green's expansion is suitably transformed, his expansion takes the form

$$
\begin{gathered}
z=x y+x^{3}+\cdots+B x^{3} y+\cdots+x^{5}+C x^{4} y \\
+\cdots+E x^{6}+F x^{5} y+\cdots+G x^{7} \\
+\cdots+I x^{8}+\cdots
\end{gathered}
$$

the omitted terms being immaterial for our purposes. The coefficients are absolute invariants of the surface. The line $x=y=0$ is the first canonical edge of Green, while the opposite edge of

* Bompiani, Per lo studio proiettivo-differenziale delle singularitd, Bollettino dell'Unione Matematica Italiana, vol. 5 (1926), p. 118.

$\dagger \mathrm{B}$. $\mathrm{Su}$, On certain quadratic cones projectively connected with a space curve and a surface, Tôhoku Mathematical Journal, vol. 38 (1933), p. 233.

$\ddagger$ Green, Memoir on the general theory of surfaces and rectilinear congruences, Transactions of this Society, vol. 20 (1919), p. 79. 
the tetrahedron of reference is Green's second canonical edge, at the origin $O(0,0,0)$. Let homogeneous coordinates be introduced by the definitions

$$
x=\frac{x_{2}}{x_{1}}, \quad y=\frac{x_{3}}{x_{1}}, \quad z=\frac{x_{4}}{x_{1}} .
$$

Then the vertex $(0,0,0,1)$ of the tetrahedron is the point, distinct from $O$, in which the first canonical edge meets the canonical quadric of Wilczynski.

The equation

$$
y=n z, \quad(n \neq 0),
$$

represents a plane through an asymptotic tangent, $y=z=0$, at the origin $O$. The plane (2) cuts the surface (1) in a curve whose projection, $C$, from the vertex $(0,0,0,1)$ onto the tangent plane, $z=0$, is represented by the equation obtained by eliminating $z$ between equations (1), (2). If this equation is solved for $y$ as a power series in $x$ the result is

(3) $y=n\left[x^{3}+n x^{4}+\left(n^{2}+1\right) x^{5}+b_{6} x^{6}+b_{7} x^{7}+b_{8} x^{8}+\cdots\right]$,

in which the coefficients $b_{6}, \ldots, b_{8}$ are defined by the formulas

$$
b_{6}=n^{3}+(B+1) n+E,
$$

(4) $b_{7}=n^{4}+(2 B+1) n^{2}+(C+E) n+G$,

$$
b_{8}=n^{5}+(3 B+1) n^{3}+(2 C+E) n^{2}+(B+F+G) n+I \text {. }
$$

The origin is obviously an inflexion on the curve $C$, and hence is also an inflexion on the curve of section, as was remarked at the outset.

3. Bompiani's Osculants at an Inflexion. The power series (3) can be employed to calculate the equations of the osculants and other lines and points associated by Bompiani with the inflexion $O$ of the curve $C$. First of all, the equation of the seven-point cusped cubic is found to be

$$
t^{2} \frac{y}{n}-l^{3}=0
$$


where $l, t$ are two linear functions defined by

$$
\begin{aligned}
& l=x+\frac{1}{12}\left(n^{2}+4\right) \frac{y}{n}, \\
& t=1-\frac{1}{2} n x-\frac{1}{2}[(B-1) n+E] \frac{y}{n} .
\end{aligned}
$$

The equation of the cusp-locus, that is, the locus of the cusps of all six-point cusped cubics, is $l=0$. The equation of the cusptangent of the seven-point cusped cubic (5) is $t=0$. The coordinates of the cusp $\mathrm{O}_{2}$ of the seven-point cusped cubic are found, by solving simultaneously the equations $l=0, t=0$, to be

$$
\left(\frac{2\left(n^{2}+4\right)}{n^{3}-4(3 B-4) n-12 E}, \frac{-24 n}{n^{3}-4(3 B-4) n-12 E}\right) \text {. }
$$

Finally, the coordinates of the point $O_{1}$ in which the cusp-tangent, $t=0$, intersects the inflexional tangent, $y=0$, of the curve $C$ are found to be

$$
\left(\frac{2}{n}, 0\right)
$$

The equation of the eight-point cubic through the cusp $\mathrm{O}_{2}$ is

$$
t^{2} \frac{y}{n}-l^{3}-U l \frac{y^{2}}{n^{2}}=0
$$

where $U$ is a polynomial defined by

$$
\begin{aligned}
U= & -\frac{1}{48} n^{4}-\frac{1}{6}(3 B-2) n^{2}+\frac{1}{2}(2 C-3 E) n \\
& +\frac{1}{3}(3 G-4) .
\end{aligned}
$$

The equation of the nine-point quartic curve which passes through the point $O_{1}$ tangent to the cusp-tangent, $t=0$, and which has a node at the point $\mathrm{O}_{2}$ with the cusp-locus, $l=0$, for one nodal tangent is

$$
t\left(t^{2} \frac{y}{n}-l^{3}-l l \frac{y^{2}}{n^{2}}\right)-V l^{2} \frac{y^{2}}{n^{2}}=0,
$$


where $V$ is a polynomial defined by

$$
V=-(C-E) n^{2}-(2 B-F+2 G-3) n-3 E+I .
$$

The equation of the residual nodal tangent of this quartic is

$$
U t+V l=0 .
$$

Finally, the equation of the line joining the origin $O$ to the intersection, distinct from $O$ and $O_{2}$, of the quartic (11) and the sevenpoint cusped cubic (5) is

$$
V^{2} \frac{y}{n}-U^{2} l=0
$$

Direct substitution from equation (3) into the left members of equations (5) and (9) leads to

$$
\begin{aligned}
& t^{2} \frac{y}{n}-l^{3}=U x^{7}+\cdots, \\
& t^{2} \frac{y}{n}-l^{3}-U l \frac{y^{2}}{n^{2}}=V x^{8}+\cdots,
\end{aligned}
$$

the omitted terms being of higher order than those written. It follows from the first of these equations that the seven-point cusped cubic (5) has eight-point contact with the curve $C$ at the point $O$ in case $U=0$. Equation (10) shows that there are four values of $n$ satisfying the equation $U=0$. Therefore through each asymptotic tangent at a point of a surface there are four planes producing sections for which the seven-point cusped cubic has eightpoint contact. For such a section the cubics (5), (9) are the same, and the quartic (11) has a cusp with the line $l=0$ for cusp tangent.

It follows from the second of equations (15) that the eightpoint cubic (9) has nine-point contact with the curve $C$ at the point $O$ in case $V=0$. Equation (12) shows that there are two values of $n$ satisfying the equation $V=0$. Therefore through each asymptotic tangent at a point of a surface there are two planes producing sections for which the eight-point cubic through the associated point $\mathrm{O}_{2}$ has nine-point contact. In this case the quartic (11) is composite. 
4. Loci of Bompiani's Osculants. The loci of Bompiani's osculants and associated lines and points for a plane section made by a variable plane through a fixed asymptotic tangent will now be considered. First of all, parametric equations for the locus of the cusp of the seven-point cusped cubic may be written by setting $x$ and $y$ respectively equal to the coordinates given in (7) and adjoining the equation $z=y / n$. Thus the locus is seen to be a twisted cubic curve. In a similar way the point $O_{1}$ is seen to generate on the tangent $y=z=0$ a point-row which is projective with the pencil of planes generated by the plane (2) and having this tangent for axis. Finally, the locus of the intersection, distinct from $O$ and $O_{2}$, of the quartic and the seven-point cubic, is a unicursal curve of order fifteen.

The equation of the locus of the cusp-locus is found by eliminating $n$ between equation (2) and the equation $l=0$, with $l$ defined by the first of (6). The result is $12 z x+y^{2}+4 z^{2}=0$. Therefore this locus is a quadric cone, as Su has shown. This cone is tangent to the tangent plane, $z=0$, along the asymptotic tangent $y=z=0$.

For the purpose of economy in writing, let us define some polynomials $p_{2}, q_{2}, r_{2}, p_{4}, p_{6}, q_{6}, r_{6}, p_{8}, p_{10}$ by the formulas

$$
\begin{aligned}
p_{2}= & z x+\frac{1}{12} y^{2}+\frac{1}{3} z^{2} \\
q_{2}= & z-\frac{1}{2} x y-\frac{1}{2}\left[(B-1) y z+E z^{2}\right] \\
r_{2}= & -(C-E) y^{2}-(2 B-F+2 G-3) y z-(3 E-I) z^{2} \\
p_{4}= & -\frac{1}{48} y^{4}-\frac{1}{6}(3 B-2) y^{2} z^{2}+\frac{1}{2}(2 C-3 E) y z^{3} \\
& +\frac{1}{3}(3 G-4) z^{4} \\
p_{6}= & p_{4} q_{2}+z^{2} r_{2} p_{2}, \\
q_{6}= & z^{2} q_{2}^{2}-p_{2}^{3}, \\
r_{6}= & z^{2} q_{2}^{2}-p_{2}^{3}-p_{4} p_{2}, \\
p_{8}= & q_{2} r_{6}-z^{2} r_{2} p_{2}^{2} \\
p_{10}= & z^{6} r_{2}^{2}-p_{4}^{2} p_{2} .
\end{aligned}
$$


Then the equation of the locus of the cusp-locus is $p_{2}=0$, as was just shown. Moreover, the equation of the locus of the cusp-tangent is $q_{2}=0$, so that this locus is a quadric surface intersecting the tangent plane, $z=0$, in the asymptotic tangents, $z=x y=0$. The plane (2) is tangent to this quadric at the point $O_{1}(2 / n, 0)$; therefore the projective correspondence mentioned above in discussing the locus of the point $O_{1}$ is the correlation between points on the tangent $y=z=0$ and the tangent planes of the quadric $q_{2}=0$ at these points. The equation of the locus of the residual nodal tangent of the nine-point quartic is $p_{6}=0$, so that this locus is an algebraic ruled surface of the sixth order, having the tangent $y=z=0$ for quintuple line and containing the other asymptotic tangent, $z=x=0$. The equation of the locus of the line joining the point $O$ to the other intersection of the nine-point quartic and the seven-point cusped cubic is $p_{10}=0$, so that this locus is an algebraic cone of the tenth order, with the tangent $y=z=0$ as ten-fold line.

The equation of the locus of the seven-point cusped cubic is $q_{6}=0$, so that this locus is an algebraic surface of the sixth order. The equation of the locus of the eight-point cubic is $r_{6}=0$, so that this locus is an algebraic surface of the sixth order. Finally, the equation of the locus of the nine-point quartic is $p_{8}=0$, so that this locus is an algebraic surface of the eighth order.

Many further properties and relations of the loci here defined can be read off immediately from their equations, but space does not permit their inclusion here.

University of Chicago 